Abstract

\title{
Genetic Engineering of Enzymes and Electrode Modifications for Biosensing Applications ${ }^{+}$
}

\author{
Lital Alfonta *, Orr Schlesinger, Itay Algov and Alon Szczupak \\ Department of Life Sciences, Ilse Katz Institute for Nanoscale Science and Technology, Ben-Gurion \\ University of the Negev, POBOx 653, Beer-Sheva 84105, Israel \\ * Correspondence: alfontal@bgu.ac.il \\ + Presented at the 5th International Symposium on Sensor Science (I3S 2017), Barcelona, Spain, \\ 27-29 September 2017.
}

Published: 22 November 2017

Biosensing efficiency, selectivity and sensitivity rely first and foremost on a successful interfacing between enzymes and sensing interfaces. An interface that allows, on the one hand, a specific analyte recognition and, on the other hand, an efficient signal transduction. Some of the challenges in biosensing stem from the incorrect orientation of the enzyme towards the sensing interface and from the need to use mediated electron transfer with a diffusional redox mediator due to difficulty in relaying a signal from a redox center that is deeply buried inside the protein matrix. Using genetic-code expansion tools, and genetic engineering approaches, we were able to modify redox enzymes and surfaces for biosensing and biofuel cell applications so they could have superior properties over native enzymes. In my talk, I will demonstrate how site-specific wiring of redox enzymes, which are genetically encoded, can improve electron transfer due to controlled and short electron transfer distances and due to proper enzyme orientation. I will also demonstrate how rational genetic engineering of an enzyme gives it superior properties for biosensing purposes compared to those of the native enzyme.

2017 by the authors. Licensee MDPI, Basel, Switzerland. This article is an open access article distributed under the terms and conditions of the Creative Commons Attribution (CC BY) license (http://creativecommons.org/licenses/by/4.0/). 